\title{
Synchronous Detection of Dual Neoplastic Malignant Disease: Adenocarcinoma of the Caecum and Renal Cell Carcinoma
}

\section{Massimo Bolognesi ${ }^{1 *}$ and Diletta Bolognesi ${ }^{2}$}

${ }^{1}$ Department of Internal General Medicine-Primary Care, ASL della Romagna-District of Cesena, Via Ungaretti 49447521 Cesena, Italy

${ }^{2}$ Department of Territorial Medicine, ASL della Romagna-District of Cesena, Via Lambruschini 30747521 Cesena, Italy

\begin{abstract}
Background: Primary cancer may occur synchronously in two different organs. The presence of a renal primary cancer discovered by chance during staging prior to surgery of another cancer (caecum-colon) is not as rare an event as is believed. In this case report the authors report an interesting case of double malignancy in which a patient was struck by two separate carcinomas, carcinoma of the caecum and renal cell carcinoma.

Case report: A 59-year-old male underwent screening with faecal occult blood test (FOBT) for colorectal cancer The resulting positive test of which required a colonoscopy indicated the malignancy of the caecum-colon. Renal cell carcinoma was detected incidentally through Computed Tomography (CT) during preliminary investigations for the staging of the disease prior to surgery. The patient subsequently underwent simultaneous, radical, right nephrectomy and hemicolectomy. Both tumours were confirmed in pathologic evaluation, revealing chromophobe renal cell carcinoma and caecum- right colon adenocarcinoma.
\end{abstract}

Conclusion: In conclusion, the elevated frequency of coexisting synchronous renal and colonic carcinomas, recommends routine use of preoperative imaging studies, in order to rule out coexistent, asymptomatic kidney malignant lesions in patients with colorectal cancer.

Keywords: Carcinoma caecum; Double malignancy; Renal cell carcinoma; Cancer patient staging

\section{Introduction}

Primary cancer may occur synchronously in two different organs. Accumulated data show infrequent yet strong association of renal cell carcinoma with multiple malignant tumours, and in particular with colon cancer [1].

According to O'Boyle \& Kemeny, 29\% of patients with renal cancer autopsy had another primary tumour, which makes it the most common tumour type of cancer associated with multiple neoplastic lesions in other organs [2].

We present an example here of pathologically documented and coexistent double malignant tumours of the colon and kidney. In the case reported, an adenocarcinoma of the caecum-colon was detect first whilst the renal tumour was discovered by chance during staging prior to surgery. Both tumours were asymptomatic and the characteristics of the renal mass (suggestive for primary tumour) excluded the possibility of being a metastasis of the other one.

The purpose of this case study was to document the accuracy of the abdominal CT scan in detecting synchronous primary malignancies, such as renal cell carcinoma, in caecum-colon cancer patients undergoing a staging workup before surgery.

\section{Case Report}

A 59-year-old asymptomatic male had positive screening for colon cancer with faecal occult blood testing (FOBT test), therefore he underwent colonoscopy. Endoscopic examination showed a vegetating, infiltrative, ulcerated lesion approximately $50 \times 30 \mathrm{~mm}$ of the caecum and ascending colon (Figure 1), which was biopsied for histological definition. An abdominal ultrasound showed a liver free from cancer lesions, but revealed a suspicious mass in the upper right kidney. The patient's haematological, renal and hepatic functions were within normal limits, as was his chest X-ray. Histological diagnosis of the colon biopsy confirmed the suspicion of colon adenocarcinoma. The patient was thus referred to the staging of the disease with an abdomen- chest computed tomography (CT) scan, in order to determine the extent of the neoplastic process. The study, gauged to be performed at all phases of contrast enhancement, indicated a heterogeneous, well-circumscribed $6.34 \mathrm{~cm}$ mass, located in the upper $1 / 3 \mathrm{right}$ kidney (Figure 2) with distributed and heterogeneous enhancement. The mass compressed the liver profile and had markedly distorted a normal calyx structure and protruded into the renal pelvis cavity. A barely thickened renal fasciae was visible in front of the mass, but there was no clear evidence of contiguity infiltration towards the descending colon. The contours of the renal peduncle were well preserved. The

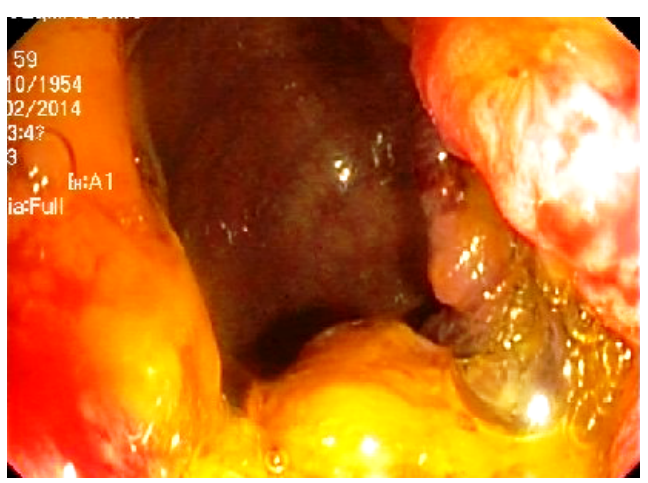

Figure 1: Colonoscopy shows a vegetating, infiltrative, ulcerated lesion approximately $50 \times 30 \mathrm{~mm}$ of the caecum and ascending colon.

*Corresponding author: Massimo Bolognesi, Department of Internal Genera Medicine, ASL della Romagna District of Cesena, Via Lambruschini 30747521 Cesena, Italy, Tel: +390547303240; E-mail: massbolo1@tin.it

Received May 15, 2014; Accepted June 28, 2014; Published June 30, 2014

Citation: Bolognesi M, Bolognesi D (2014) Synchronous Detection of Dual Neoplastic Malignant Disease: Adenocarcinoma of the Caecum and Renal Cell Carcinoma. J Clin Case Rep 4: 377. doi:10.4172/2165-7920.1000377

Copyright: (c) 2014 Bolognesi M, et al. This is an open-access article distributed under the terms of the Creative Commons Attribution License, which permits unrestricted use, distribution, and reproduction in any medium, provided the original author and source are credited. 


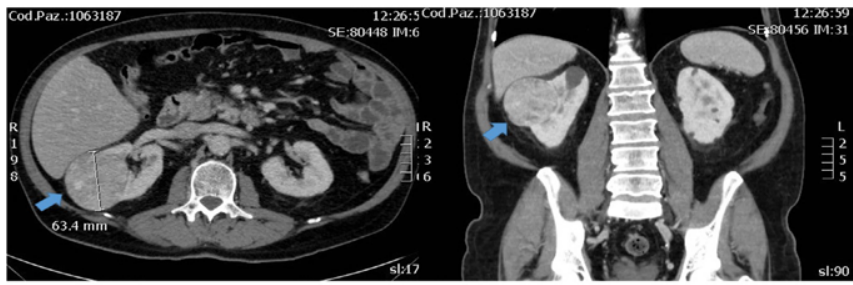

Figure 2: Computed Tomography (CT or CAT) Scan of the Abdomen shows a well-circumscribed $6,34 \mathrm{~cm}$ mass, located in the upper $1 / 3$ of the right kidney (see blue arrows).
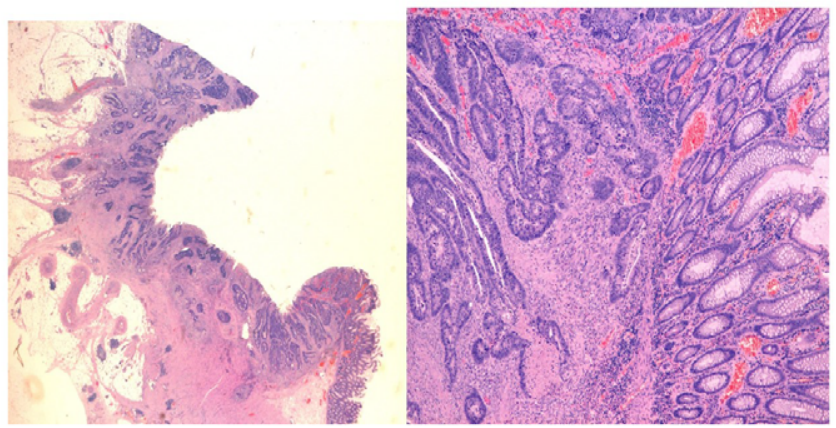

Figure 3: Histopathology specimen showing adenocarcinoma of the caecum and ascending colon.

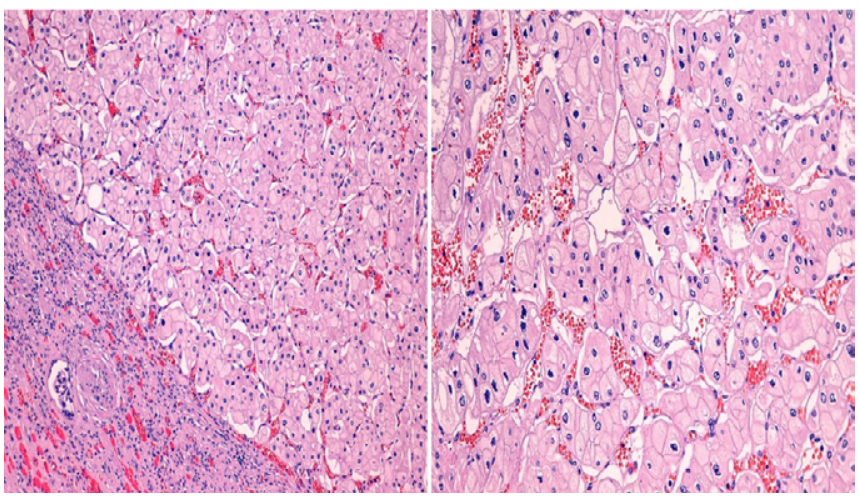

Figure 4: Histopathology specimen showing chromophobe renal cell carcinoma.

lesion appeared to be vascularized very well, having a strong arterial phase contrast media enhancement, with imaging pictures being indicative of renal carcinoma. Surgery, subsequently confirmed a large, ulcerative and infiltrative lesion in the caecum-ascending colon area with a few swollen regional lymph nodes for which classical right hemicolectomy was performed. Intestinal anastomosis small intestine large intestine was done, with contemporary radical right nephrectomy for the kidney mass considered as being a malignant tumour. Section cutting of the samples, bowel resection and nephrectomy revealed gross characteristics of adenocarcinoma of the caecum and renal cell carcinoma (RCC). Histopathological examination confirmed highgrade infiltrating adenocarcinoma of the ascending colon and ileocecal valve (Figure 3), and chromophobe renal cell carcinoma (Figure 4), respectively, with no evidence of lymph node metastases and resection margins free of neoplastic disease. The patient was discharged 8 days following surgery in good physical condition.

\section{Discussion}

Since Billroth published his first report of multiple primary malignancy in 1889 [3], numerous reports of multiple carcinomas of the large gut, arising simultaneously or as interval carcinomas, have been published [4]. The occurrence of multiple neoplasms is not rare. The Second Primary Malignancy (SPM) is a second de novo malignant neoplasm occurring in a patient with recognized cancer [5]. Most multiple primary cancers are double primary cancers, and the incidence has decreased as the number of concomitant cancers has increased [6]. Some case reports of synchronous colon and renal cancer are published in literature [7-10]. Certain criteria have been set down to diagnose double malignancy. In order to make the diagnosis of a second primary malignant neoplasm, each tumour should be separate from the other, each should be malignant, and neither should be a metastasis from the other, i.e., microscopic and morphologic features of the two tumours must be entirely different [11] The coincidental detection of one or more additional primary tumours at the CT staging of a patient with a known malignancy is a more frequent occurrence for physicians dealing with malignant neoplasm and their staging [12]. Detection of a synchronous, second tumour poses several problems for differential diagnosis and cancer patient management. Our case showed clinical features primarily because od adenocarcinoma of the caecum and coexistence with other primary neoplasm, i.e., RCC that was diagnosed on CT scan of abdomen during workup staging. Therefore, the "Warren and Gates Criteria for Diagnosis of Multiple Primary Malignancies" is confirmed:

1. Each of the tumours must be malignancy confirmed by histology

2. Each one must be geographically separate and distinct. The lesions should be separated by normal mucosa.

3. The probability of one being the metastasis of the other must be excluded.

Treatment strategies in cases of double malignancy depend on first treating the malignancy that is more advanced, or sometimes, both malignancies might be treated simultaneously, if therapeutic option is the same for both $[13,14]$.

In our case, both diseases were surgically amenable and treated by carrying out right hemicolectomy and omolateral nephrectomy in a single session. Primary tumour can occur in a synchronous manner in two different organs, in which case simultaneous resection is recommended, if possible. To our knowledge this is one of the original few cases described in the literature of simultaneous surgical removal of primary double malignancies coexisting both in kidney and colon [5]. As Bhargava et al. [15] reported in their work, this report confirmed the importance of staging work-up in all cancer cases, especially because the coexistence of renal cell carcinoma together with other primitive malignancies is very frequent [16].

\section{Conclusion}

The teaching point of this case report highlighted the crucial importance of staging of disease prior to surgery, which allowed the incidental discovery of another primary malignancy and permitted radical excision, resulting in a better prognosis for the patient. Finally, the relative frequency and incidence of synchronous kidney and colon carcinomas, recommend routine use of preoperative imaging studies to rule out coexisting asymptomatic malignant lesions of the kidney in patients with colorectal cancer, and how this can impact on the further management of these patients. With the advent of new diagnostic 
Citation: Bolognesi M, Bolognesi D (2014) Synchronous Detection of Dual Neoplastic Malignant Disease: Adenocarcinoma of the Caecum and Renal Cell Carcinoma. J Clin Case Rep 4: 377. doi:10.4172/2165-7920.1000377

techniques for the staging of the disease as well as advances in the management of common cancer, the detection of SPM has increased. This permits the majority of the malignancies diagnosed simultaneously to be removed in one single step.

\section{Acknowledgement}

The authors thanks Paolo Pazzi, Maurizio Mughetti and Evandro Nigrisoli for their courtesy to have provided images of the clinical case.

\section{References}

1. Amoroso A, Del Porto F, Garzia P, llardi M, Clemenzia C, et al. (1999) The infrequent association of synchronous renal and colonic malignancies. Eur Rev Med PharmacolSci 3: 111-114.

2. O'Boyle KP, Kemeny N (1989) Synchronous colon and renal cancers: six cases of a clinical entity. Am J Med 87: 691-693.

3. Billroth $\mathrm{H}$, Die Allgemeire, Chirurgie (1952) Multiple primary malignant tumours involving the colon and rectum. Am J Surg 55: 83-85.

4. Boice JD, Storal M, Bletter M (1992) Cancer in contralateral breast after radiotherapy for breast cancer. $\mathrm{N}$ Engl J Med 327: 431-34.

5. Hulika N, Ray S, Thomas J, Fernandes DJ (2012) Second primary malignant neoplasms: a clinicopathological analysis from a cancer centre in India. Asian Pac J Cancer Prev 13: 6087-6091.

6. Cheng HY, Chu CH, Chang WH, Hsu TC, Lin SC, et al. (2005) Clinical analysis of multiple primary malignancies in the digestive system: a hospital-based study. World J Gastroenterol 11: 4215-4219.
7. DalaS, Garg P, Nityasha, Jain A (2006) Synchronous double malignancy: Adeno-Carcinoma of Caecum and Renal Cell Carcinoma. The Internet Journal of Gastroenterology 6 .

8. Luczynska E, Pawlik T, Aniol J, Chwalibóg A (2008) Synchronous colon and renal cancer-case report Pol J Radiol. 73:78-80.

9. Kim SH, Park JY, Joh YG, Hoe HE (2004) Simultaneous laparoscopic radical nephrectomy and laparoscopic sigmoidectomy for synchronous renal cell carcinoma and colonic adenocarcinoma. J Laparoendosc Adv Surg Tech A 14:179-81.

10. Dalli J, Camilleri-Brennan J (2011) Synchronous colonic adenocarcinoma and renal oncocytoma: a case report and literature review. Scott Med J 56: 181.

11. Kishimoto T, Hashimoto H, Ono T, Okada K (1992) Synchronous double malignancy: adenocarcinoma of lung and malignant astrocytoma induced by asbestos exposure. Cancer Invest 10: 129-133.

12. Catalano O, Petrillo M, Nunziata A, Fusco R, Carone G, et al. Synchronous tumours detected during cancer patient staging: prevalence and patterns of occurrence at MDCT; ECR 2013/C-1607.

13. Warren S, Gates O (1932). Multiple primary malignant tumors: A survey of the literature and statistical study. Am J Cancer 16: 1358-414.

14. Agrawal R (2007) Synchronous dual malignancy: successfully treated cases. J Cancer Res Ther 3: 153-156.

15. Bhargava A, O'Callaghan M, Abdelhafiz T, Downey P, Nasr A, et al. (2012) Synchronous sigmoid and caecal cancers together with a primary renal cell carcinoma. Ir J Med Sci 181: 273-276.

16. Beisland C, Talleraas O, Bakke A, Norstein J (2006) Multiple primary malignancies in patients with renal cell carcinoma: a national population-based cohort study. BJU Int 97: 698-702. 